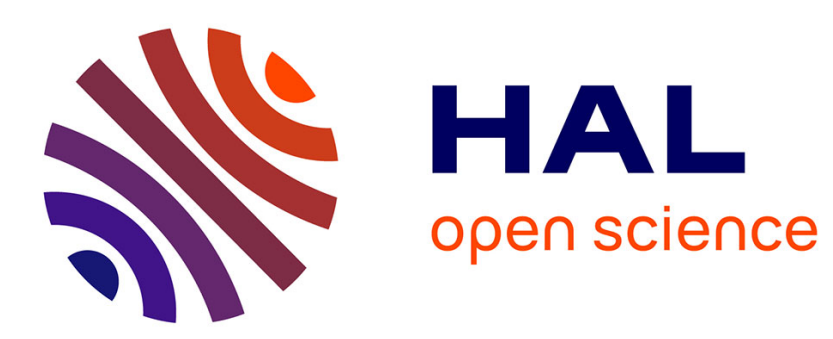

\title{
Du terrain à la buvette: diffusion du football et contrôle social en région parisienne dans l'entre-deux-guerres
}

\author{
Julien Sorez
}

\section{To cite this version:}

Julien Sorez. Du terrain à la buvette: diffusion du football et contrôle social en région parisienne dans l'entre-deux-guerres. Le Mouvement social, 2012, Sociabilités urbaines et contrôle social, 1 (238), p.65-80. 10.3917/lms.238.0065 . hal-02559472

\section{HAL Id: hal-02559472 \\ https://hal.science/hal-02559472}

Submitted on 30 Apr 2020

HAL is a multi-disciplinary open access archive for the deposit and dissemination of scientific research documents, whether they are published or not. The documents may come from teaching and research institutions in France or abroad, or from public or private research centers.
L'archive ouverte pluridisciplinaire HAL, est destinée au dépôt et à la diffusion de documents scientifiques de niveau recherche, publiés ou non, émanant des établissements d'enseignement et de recherche français ou étrangers, des laboratoires publics ou privés. 


\title{
DU TERRAIN À LA BUVETTE : DIFFUSION DU FOOTBALL ET CONTRÔLE SOCIAL EN RÉGION PARISIENNE DURANT L'ENTRE-DEUX-GUERRES
}

\author{
Julien Sorez \\ La Découverte | Le Mouvement Social
}

\begin{abstract}
$2012 / 1-n^{\circ} 238$
pages 65 à 80
\end{abstract}

ISSN 0027-2671

Article disponible en ligne à l'adresse:

http://www.cairn.info/revue-le-mouvement-social-2012-1-page-65.htm

Pour citer cet article :

Sorez Julien, « Du terrain à la buvette : diffusion du football et contrôle social en région parisienne durant l'entre-deux-guerres »,

Le Mouvement Social, 2012/1 n²38, p. 65-80. DOI : 10.3917/Ims.238.0065

Distribution électronique Cairn.info pour La Découverte.

(c) La Découverte. Tous droits réservés pour tous pays.

La reproduction ou représentation de cet article, notamment par photocopie, n'est autorisée que dans les limites des conditions générales d'utilisation du site ou, le cas échéant, des conditions générales de la licence souscrite par votre établissement. Toute autre reproduction ou représentation, en tout ou partie, sous quelque forme et de quelque manière que ce soit, est interdite sauf accord préalable et écrit de l'éditeur, en dehors des cas prévus par la législation en vigueur en France. II est précisé que son stockage dans une base de données est également interdit. 


\title{
Du terrain à la buvette : diffusion du football et contrôle social en région parisienne durant l'entre-deux-guerres
}

\author{
par Julien SorEz ${ }^{*}$
}

$\mathrm{T}$ héâtre d'un spectacle devenu central de la culture de masse, le stade est un lieu d'expression de l'émotion collective et des " passions partisanes " ${ }^{1}$. À ce titre, il est un espace privilégié de l'observation des mécanismes de la violence collective, des modifications de la consommation culturelle ou encore de la manipulation des foules $^{2}$. Si le stade a alimenté un certain nombre de travaux en sciences sociales, la recherche en histoire reste encore en retrait de l'analyse d'un espace pourtant extrêmement sensible aux évolutions des sociétés qui l'ont bâti et investi. Les études consacrées aux enceintes sportives appréhendent et donc consacrent le stade comme un produit des politiques publiques de l'entre-deux-guerres, qu'elles soient mises en œuvre par les municipalités tercio-républicaines ou par les chefs des États autoritaires européens ${ }^{3}$. L'influence d'Alain Ehrenberg sur ces travaux se mesure à la place centrale qu'occupe l'analyse des éléments architecturaux, supposés traduire dans la pierre les principes qui structurent ces régimes ou les ambitions personnelles de leurs commanditaires ${ }^{4}$. Toutefois, cette propension à valoriser des espaces conçus et aménagés par des pouvoirs politiques n’épuise pas les enjeux de la construction et de la multiplication des enceintes sportives. Pour notre part, nous considérons que les dispositifs architecturaux, la monumentalité des enceintes sportives ou la mise en œuvre d'une politique d'équipements sportifs soulignent davantage les intentions des acteurs que l'impact politique et idéologique de leurs réalisations, et qu'il convient donc de ne pas prendre pour acquis les effets supposés de la mise en scène ou d'une démarche programmatique ${ }^{5}$.

* Agrégé et docteur en histoire, ATER à l'IEP de Paris.

1. C. Bromberger, Le match de football. Ethnologie d'une passion partisane à Marseille, Naples et Turin, Paris, Éd. de la Maison des Sciences de l'Homme, 1995.

2. On pourra se reporter aux deux contributions les plus influentes: A. EhrenberG, « Aimez-vous les stades? Architecture de masse et mobilisation ", in A. EHrenberg (éd.), Aimez-vous les stades? Les origines historiques des politiques sportives en France (1870-1930), Recherches, ${ }^{\circ} 43,1980$, p. 25-54; N. Elias et E. Dunning, Sport et civilisation. La violence maîtrisée, Paris, Fayard, 1994, notamment les chapitres 8 et 9. Parmi les travaux sociologiques, on pourra consulter : W. Nuytens, "Le supporter de football et la règle : entre la faire et la défaire ", Déviance et société, vol. 29, 2005/2, p. 155-166 ; P. Mignon, "Faire corps : Supporters ultra et hooligans dans les stades de football ", Communications, n67, 1998, p. 47-58; N. HourCade, "La place des supporters dans le monde du football », Pouvoirs, nº101, avril 2002, p. 75-87.

3. É. Lê-Germain, "La cité des sports à Lyon : fleuron architectural et symbole politique (19101931) ", in P. Dufieux (dir.), Tony Garnier. La cité industrielle et l'Europe, Lyon, Conseil d'Architecture, d'Urbanisme et de l'Environnement, 2009, p. 202-213 ; P. Arnaud (dir.), «Le Sport et la Ville », Spirales, $\mathrm{n}^{\circ} 5$, Lyon, Centre de Recherches et d'Innovation sur le Sport, 1992 ; D. Bolz, Les arènes totalitaires. Fascisme, nazisme et propagande sportive, Paris, CNRS éditions, 2008.

4. A. Ehrenberg, "Aimez-vous les stades ?..." art. cité.

5. Il faudrait pour cela mobiliser des corpus documentaires qui permettent de mesurer les effets réels de ces réalisations sur les usagers de ces enceintes. 
Afin d'enrichir les perspectives de recherche héritées des travaux précédents, nous sommes amené à reconsidérer la nature du regard que les chercheurs ont jusque-là porté sur cet objet d'étude. Tout d'abord, la démarche qui compare les politiques sportives à l'échelle des États ou des municipalités, pour pertinente qu'elle soit, ne doit pas évacuer la diversité des acteurs que la multiplication des enceintes sportives nous permet d'observer. Aux côtés des pouvoirs publics, les associations sportives, les entreprises ou encore les patronages catholiques participent également à la construction de stades dans l'entre-deux-guerres, notamment dans des pays comme la France où le rôle de l'État fut alors beaucoup plus mesuré. Cette attention à la multiplicité des acteurs permet notamment d'intégrer au champ d'étude des espaces de la pratique amateur et anonyme. Aussi, l'étude à grande échelle des complexes sportifs doit-elle prendre en considération tous les lieux qui composent l'ensemble du dispositif spatial (terrains, vestiaires, tribunes, squares, buvettes, etc.), pour mesurer leur interdépendance et tenter de mettre en évidence les usages sociaux qu'ils impliquent. Car si l'étude des espaces dédiés à la pratique sportive révèle leur visée idéologique et leur instrumentalisation politique, il nous paraît essentiel de saisir la dynamique sociale que ces différents lieux contribuent non seulement à intégrer mais aussi à forger. Enfin, le regard historique ne doit pas perdre de vue que les enceintes pérennes et les pratiques sportives qui les habitent sont dans une relation d'interférence. Structures souvent omnisports en France, les stades sont " pratiqués " par des usagers qui, en fonction de leur origine sociale, de leur discipline sportive, redéfinissent les lieux qu'ils occupent. En retour, les pratiques sportives, codifiées par un cadre réglementaire établi par les institutions sportives compétentes, se définissent aussi dans leur interaction avec les espaces dans lesquels elles évoluent.

Ainsi, l'étude du stade de football à l'échelle de la région parisienne, en intégrant un nombre conséquent d'enceintes et les espaces attenants au terrain proprement dit, représente un point d'observation privilégié du processus de domestication des individus opéré par les différents acteurs du monde sportif tels que les patronages catholiques, les entreprises ou encore les municipalités du département de la Seine. Cette mainmise complexe et subtile ne se fonde pas seulement sur une contrainte extérieure que manifestent des règlements et une relation contractuelle entre les usagers et les institutions qui gèrent les différentes installations sportives, mais elle nous montre la manière dont les stades et donc le sport participent à l'intériorisation des normes sociales dans la France de l'entre-deux-guerres.

\section{Du café à la buvette : la capture d'une sociabilité sportive}

De la fin du XIXe siècle au milieu des années 1920, les associations sportives du département de la Seine éprouvent les pires difficultés pour s'établir durablement dans l'espace urbain ou périurbain. L'étroitesse de leurs ressources financières, la pression foncière liée à la forte urbanisation et à l'industrialisation ainsi que le peu d'intérêt des élus locaux à l'égard du mouvement sportif, expliquent le nomadisme de la grande majorité des clubs. Cette situation concerne à la fois les terrains de jeu, dont la jouissance ne dure jamais très longtemps, mais aussi les locaux associatifs dans lesquels les joueurs peuvent se changer et les dirigeants se réunir ${ }^{6}$. Dans ces

6. Sur les conditions précaires des premières équipes de football, on se reportera à J. SoREZ, « Le football et la fabrique des territoires. Une approche spatiale des pratiques culturelles », Vingtième Siècle. Revue d'histoire, 2011/3, n'111, p. 59-72. 
conditions, les débits de boisson, cafés et autres commerces de bouche jouent un rôle de premier plan. D'une part, ils abritent le jour du match les joueurs pour qu'ils puissent se changer et remiser leurs effets personnels et le matériel comme les poteaux des buts qu'il convient d'installer et de démonter à chaque rencontre. Ces " cafés vestiaires " se situent souvent à proximité des terrains de jeu et n'offrent aucun confort aux usagers qui se plaignent de l'accueil qui leur est réservé. Aussi les cafés servent-ils de point d'ancrage à l'existence administrative des clubs. En effet, nombre d'entre eux constituent les sièges sociaux des associations sportives et mettent à disposition des dirigeants un espace privatif, lorsque la configuration du lieu l'autorise. Lieu de prise de décision, le café est surtout le cœur de la sociabilité sportive. Que ce soit au cours des réunions des commissions sportives ou du comité, des soirées organisées pour fédérer des clubs composés de plusieurs équipes et pour cultiver les liens entre joueurs et dirigeants, ou pour abriter les discussions d'avant et d'après match qui rythment les fins de semaine, le café permet aux membres et sympathisants de l'association de se retrouver. Lieu de prédilection des parties de cartes ou de billard entre les adhérents du club, il a par la consommation d'alcool et les soirées tabagiques vocation à cultiver l'entre-soi d'une pratique exclusivement réservée aux hommes jusqu'à la Première Guerre mondiale ${ }^{7}$, comme le suggèrent les propos des dirigeants du Racing Club de France à la fin des années 1900 :

"Et voilà pourquoi, samedi dernier, beaucoup de joueurs d'association, des joueurs de cricket, quelques rugbymen, se sont réunis chez Mollard, dans les salons du premier étage. Après que notre ami Lejeune nous eut rappelé pourquoi nous étions là, nous nous sommes mis à bavarder de tout un peu, mais du Racing surtout, nous avons fumé, - ça, c'était obligatoire et c'est pour cela que les dames n'étaient pas admises, - et de temps en temps, jusqu'à minuit, nous eûmes le plaisir d'entendre chanter l'un, chanter l'autre, d'écouter le piano, d'entendre le violon, de rire aux éclats sous les bons mots de monologues parfaitement récités, de voir l'un de nos camarades projeter sur un écran et d'un fusain habile et rapide, les caricatures de quelques-uns d'entre nous ${ }^{8}$.

S'il représente un espace par lequel les dirigeants de l'association tentent de garder l'emprise sur leurs membres actifs, rares sont les cafés qui abritent durablement une association sportive. Le changement de l'équipe dirigeante, la migration vers un autre terrain de jeu nous rappellent que le lien qui les unit à l'association demeure fragile. Une partie du monde dirigeant cultive à l'égard du café une certaine défiance dans la mesure où la consommation d'alcool et la tabagie qui accompagnent la sociabilité associative vont à l'encontre de la portée hygiéniste de la pratique sportive. Pour preuve, la plaquette imprimée par l'Union des sociétés sportives et gymniques du travail (USSGT) dans les années 1920 afin de guider les premiers pas des dirigeants de la nouvelle fédération socialiste en leur expliquant " comment former un club de l'USSGT ", conseille à ses membres, sous la rubrique "Organisation ", une fois l'association constituée, de " chercher le plus tôt possible une salle pour la culture physique ou la gymnastique et pour les réunions " en prenant soin d' " éviter la salle

7. M. Agulhon, Le cercle dans la France bourgeoise, 1810-1848. Étude d'une mutation de sociabilité, Paris, Armand Colin, 1977, et A.-M. SoHN, Sois un homme! La construction de la masculinité au XIX' siècle, Paris, Seuil, 2009.

8. Tous les sports, organe officiel de l'Union des Sociétés Françaises de Sports Athlétiques, 12 novembre 1909. 
de café $"{ }^{9}$. D'ailleurs, la plupart des clubs sportifs qui reçoivent l'aide d'une municipalité socialiste ou communiste dans l'entre-deux-guerres ont pour siège la mairie de leur commune comme l'Union Sportive du Travail d'Ivry, la Jeunesse Sportive de L'Haÿ-les-Roses, ou encore le Club Athlétique Ouvrier de Villejuif dont le siège était jusqu'en 1925, date de la victoire communiste aux élections municipales, un débit de boisson de la localité appartenant à son vice-président. L'association travailliste remplace à la fin des années 1920 l'Étoile Sportive de Villejuif, club affilié à la Fédération Française de Football, qui bénéficiait de la bienveillance édilitaire jusqu'alors ${ }^{10}$.

Ainsi, le café accompagne le développement du mouvement sportif durant plus de trois décennies. Mais contrairement à la Grande-Bretagne, où il est un acteur de premier plan dans la diffusion sociale du football par la location de terrains aux joueurs, la mise sur pied d'équipes composées de clients ou l'organisation de paris sportifs ${ }^{11}$, ces espaces de sociabilité restent éphémères et ne portent que rarement l'identité du club ${ }^{12}$. Avec la pérennisation des installations sous la forme de stades ou de terrains de sports clôturés dont profite le football à partir des années 1910 et surtout durant l'entre-deux-guerres, la place des cafés décroît progressivement au profit d'un autre espace de sociabilité, la buvette. Alors que se multiplient les enceintes sportives patronales et municipales dans les communes de Seine banlieue et de Seine-et-Oise, on remarque l'installation systématique de buvettes au sein du dispositif spatial du stade ou du complexe sportif. Les patronages catholiques parisiens disposant d'un local dans leur paroisse ont été les premiers à ouvrir une buvette afin de remédier à l'influence perçue comme néfaste des cafés. Ainsi, dès 1909, l'Union Sportive d'Auteuil installe une buvette au patronage et, " dans l'intérêt de la discipline et de la bonne tenue, il est défendu aux équipes de faire usage des buvettes étrangères " ${ }^{13}$. De même, le règlement du Patronage Olier pendant la Grande Guerre atteste l'existence d'une buvette dont la responsabilité échoit au concierge du terrain de la Vache Noire situé à Montrouge :

"Mme Bourdais tient à la disposition des joueurs de football, sous le contrôle du directeur du patronage, une buvette où l'on peut trouver les consommations suivantes, à l'exclusion de tout alcool, liqueur, etc. : grog chaud, sirop, vin blanc, chocolat, sandwich. Le principal avantage de cette Buvette est de permettre aux membres du Patronage de se grouper au Patronage après les jeux et de les empêcher d'aller dans les cafés voisins " ${ }^{14}$.

La création d'un espace de sociabilité intégré au dispositif spatial du stade et donc coupé du monde extérieur, se généralise avec la multiplication des stades et complexes sportifs municipaux dans l'entre-deux-guerres. L'article $34 \mathrm{du}$ règlement du Stade municipal du Perreux stipule que " la buvette à l'intérieur du stade ne

9. Archives municipales (AM) Puteaux, 4R 1, "Correspondance des associations sportives ».

10. Archives départementales (AD) Val-de-Marne, 1807W 38, "Club Athlétique Ouvrier de Villejuif".

11. T. Mason, Association Football and English Society, 1863-1915, Brighton, Harvester Press ; Atlantic Highlands (New Jersey), Humanities Press, 1980, plus particulièrement le chapitre 2.

12. Si l'on considère que le nom des cafés et commerces de bouche nous indique l'existence d'une volonté de cadrer avec leur clientèle d'habitués, force est de constater que très peu de commerces s'octroient dans le département de la Seine une identité sportive.

13. Pages d'Auteuil, bulletin du Cercle Jeanne d'Arc et de l'Union Sportive d'Auteuil, 10 octobre 1909.

14. Instructions concernant le Patronage Olier 1914 (manuscrit), "Règlement à suivre pendant la guerre $1914 »$. 
vendra que des boissons hygiéniques telles que bière, vin, limonade, sirops, café, thé, consommés, lait, eaux minérales, etc. ${ }^{15}$. Le contrôle de la consommation de boissons au stade par la municipalité est aussi de rigueur dans le cahier des charges rédigé en vue d'une adjudication publique par les élus de Châtillon. Le concessionnaire, qui doit verser un cautionnement de 3000 francs, et fournir tout le matériel nécessaire " à un service convenable et rapide ", du comptoir avec l'appareil percolateur au service complet, est obligé d'afficher de manière " très apparente " le contenu des consommations que le cahier des charges détaille avec une grande précision ${ }^{16}$. Les prix sont fixés par la municipalité ainsi que la liste des boissons autorisées qui, à part le rhum, le kirsch et le cognac, propose surtout un large choix de vins, bières et mousseux. Proposer, comme au patronage, un espace de consommation de boissons procède explicitement de la volonté de soustraire les joueurs et les spectateurs aux effets de l'ébriété et d'édifier un espace alternatif aux cafés. L'obligation d' "ouvrir tous les dimanches et jours fériés et pour toutes les manifestations organisées sur le stade " montre que l'enjeu est bien de réorienter la clientèle sportive vers un lieu aux mœurs soigneusement contrôlées puisque " le service sera assuré de préférence par du personnel masculin et de bonne tenue. Tout racolage des consommateurs par une réclame indiscrète ou tapageuse est rigoureusement interdit ${ }^{17}$. Lorsque le chiffre d'affaires de la buvette est insuffisant, la municipalité de Châtillon consent à prolonger gratuitement le bail pour éviter que ne disparaisse cet espace de sociabilité sous contrôle ${ }^{18}$. Ce même impératif est souligné par la demande de M. Delarbre qui souhaite qu'une concession puisse être accordée à un cafetier dans le parc de La Courneuve car, " après leur partie, les jeunes gens ont besoin d'un sandwich et une boisson chaude. Tout cela à des prix acceptables ${ }^{19}$.

La volonté de trouver une alternative aux cafés est renforcée par l'aménagement de vestiaires. Les installations sportives intègrent en effet des espaces consacrés aux soins du corps et à la remise des effets personnels des sportifs. Dès 1913, les dirigeants du Club Athlétique de Vitry, font établir à proximité du terrain qu'ils viennent d'acquérir " un coquet pavillon comprenant vestiaire et buvette " ${ }^{20}$. La visite guidée du stade municipal de Thiais, réalisée par un journaliste de Banlieue Sud avant l'inauguration, montre l'attraction que représente ce type d'équipement : "Que voyons-nous, évidemment un superbe ensemble, mais tout d'abord à notre gauche, à quelques pas, un élégant pavillon en briques rouges, il est propre, il est coquet, visitons-le... C'est là, voyez-vous, dans ses sous-sols que seront installés, largement aérés, de confortables vestiaires, dotés de commodités indispensables, douches, water, etc. ${ }^{21}$. Quant aux installations du stade du club sportif de la revue L'Illustration à Bobigny, " les vestiaires sont chauffés et les douches réparatrices » 22

15. AM Le Perreux, 7D 2/20, réunion de la commission du stade du Perreux du 17 mars 1934.

16. AM Châtillon, 1D 20, conseil municipal, délibération du 29 novembre 1935.

17. Ibid.

18. Ibid., délibération du 28 décembre 1934.

19. AM La Courneuve, 4R 1, Parc des Sports, séance de la sous-commission du Parc des Sports de La Courneuve du 17 octobre 1933.

20. L'Auto, 12 septembre 1913.

21. Banlieue Sud, organe hebdomadaire d'information, édition Choisy-le-Roi-Saint-Maur, 30 septembre 1933 .

22. L'Auto, 17 décembre 1934. 
alors que celles du stade de la Société Athlétique de Valmy, à Maisons-Alfort, bénéficient d'une salle d'hydrothérapie ${ }^{23}$.

Par conséquent, la prise en charge des deux fonctions essentielles du café, le contrôle effectué sur les boissons et la tenue du lieu, laisse à penser que la buvette a pour fonction de détourner les usages sportifs des cafés. Cette récupération est orchestrée par une partie du monde dirigeant, préoccupée par l'éducation morale des membres actifs, à l'image des directeurs de patronages ou des élus municipaux pour qui le sport représente un pan essentiel de la politique hygiéniste. La mise sous contrôle de la sociabilité sportive par la création d'espaces dont les fonctions se substituent à celles des cafés n'est toutefois pas spécifique aux organisations sportives. Dès le début du XX $\mathrm{XX}^{\mathrm{e}}$ siècle, les responsables politiques et syndicaux, après avoir utilisé les cafés comme espaces privilégiés de réunion et de mobilisation, délaissent, à mesure que leur mouvement s'institutionnalise, ces espaces de sociabilité au profit des Bourses du travail ou des sièges institutionnels ${ }^{24}$. Mais si elle traduit dans le cadre politique et syndical un repli de l'organisation sur elle-même, elle illustre au sein des clubs sportifs une emprise croissante du monde dirigeant, associatif, patronal et politique sur leurs membres actifs. Cette évolution ne suffit pourtant pas à éradiquer le café de l'univers de référence des sportifs. Au moment où les stades patronaux, associatifs et municipaux proposent des espaces concurrentiels, la spectacularisation puis la professionnalisation du football contribuent à maintenir l'image du café comme lieu de prédilection de la sociabilité sportive. Alors qu'il permettait de réunir dans un même lieu dirigeants et membres actifs que les exigences de la compétition dispersaient aux quatre coins de la région, le café devient une interface entre les joueurs et les supporters du club qui apparaissent progressivement au cours des années 1910 et se généralisent dans les années $1920^{25}$. Son importance dans les représentations collectives des acteurs du monde du football peut aussi se mesurer à la place de choix qu'il occupe dans les stratégies de reconversion des joueurs pour qui l'achat et la tenue d'un café est un signe de promotion sociale ${ }^{26}$.

\section{Stade, famille et " petite patrie " : les ressorts de la domination sociale}

Dans la Seine de l'entre-deux-guerres, de nombreuses entreprises mettent des stades et complexes sportifs à la disposition de leurs employés. À Bobigny, après l'Union Sportive des Galeries Lafayette, nous avons évoqué l'aménagement par L'Illustration d'un stade pour ses employés qui jouent au football et au basket sous les couleurs de l'Illustration sportive ${ }^{27}$. À Maisons-Alfort, l'industriel vinicole Nicolas fait construire en 1935 un stade éponyme pour ceux de ses employés qui pratiquent le football dans le cadre de la Société Athlétique de Valmy. La description qu'en donne L'Auto,

23. Ibid., 13 janvier 1935.

24. N. Graveleau, Les cafés comme lieux de sociabilité politique à Paris et en banlieue 1905-1913, maîtrise d'histoire, Université Paris 1-CRHMSS, 1991.

25. Le café de Rome près de la gare Saint-Lazare est par exemple le siège du club en même temps qu'il devient le point de ralliement des supporters du Club Français à partir de la Première Guerre mondiale. Cf. Bulletin de Guerre du Club Français, février 1919.

26. A. Wahl et P. Lanfranchi, Les footballeurs professionnels des années 1930 à nos jours, Paris, Hachette, 1995, p. 152-155.

27. L'Auto, 17 décembre 1934. 
systématiquement élogieuse à l'égard des œuvres sportives patronales, est édifiante. La construction du stade est présentée comme l'initiative du directeur administratif et sportif de l'établissement, Le Bour, parce que les employés de Nicolas membres de la S. A. de Valmy étaient auparavant « molestés par les gardes du Bois [de Vincennes], et furent souvent l'objet de plaintes et de contraventions qui décourageaient les jeunes sportifs traqués par les gardiens " ${ }^{28}$. Le contrôle social exercé par le moyen de ces installations ne fait aucun doute :

«C'est donc dans un esprit de famille et de grande camaraderie que l'on travaille dans ces vastes établissements de Charenton et c'est avec cette même saine mentalité que les athlètes font du sport. Celui-ci [Nicolas] a compris que le sport est un bienfait social qui resserre les liens d'amitié qui doivent exister entre l'employeur et l'employé et que, pour avoir un personnel de choix, il faut bien lui faire de la santé, et c'est le sport qui s'en charge ${ }^{29}$.

Cette bienveillance patronale est aussi soulignée à l'occasion de la présentation du stade d'Enghien-Ermont ${ }^{30}$ aménagé par la direction de la Compagnie du Nord pour les employés et les ouvriers des ateliers des chemins de fer du Nord : "Ces installations ne leur donnent pas seulement toutes les facilités pour la pratique de leur sport favori, mais leur procurent également un délassement moral. Car le stade d'EnghienErmont constitue une grande famille. On n'y cherche pas à faire des champions, des phénomènes, mais on se borne à permettre à la masse de s'exercer afin d'acquérir de la santé, de la vigueur ${ }^{31}$. De telles réalisations traduisent la volonté patronale d'investir le champ des loisirs de leurs employés et de cultiver, au nom de la prise en charge de leur bien-être, un paternalisme qui se caractérise par un " contrôle total " de l'individu ${ }^{32}$. Dans cette perspective, le stade n'est qu'une pièce supplémentaire au dispositif d'encadrement des employés et ouvriers.

Mais la mobilisation de la référence à la famille ou à l'esprit de famille n'est pas exclusivement une métaphore pour qualifier la camaraderie que souhaite imposer le patronat à ses employés. Dans certains cas, elle implique une réelle tentative d'ouverture du recrutement des usagers de ces équipements sportifs. Les dirigeants du Club Athlétique de la Société Générale construisent un square à proximité du stade Minvielle ${ }^{33}$ " pour permettre aux mamans et à leurs enfants de bénéficier du bon air du Bois de Boulogne et de toute la tranquillité d'un stade fermé " ${ }^{34}$. Même chose au complexe de l'Association Sportive de la Bourse à Vaucresson, où l'étendue

28. Ibid., 13 janvier 1935.

29. Ibid.

30. Enghien et Ermont, tout comme Vaucresson, se trouvent en Seine-et-Oise.

31. L'Auto, 13 août 1929.

32. G. NoIRIEL, «Du "patronage" au "paternalisme" : la restructuration des formes de domination de la main-d'œuvre ouvrière dans l'industrie métallurgique française ", Le Mouvement Social, $n^{\circ} 144$, juillet-septembre 1988, p. 17-35. Le confort des installations d'entreprise s'inscrit par exemple dans cette volonté du patronat de conserver sa main-d'œuvre.

33. Louis Minvielle est un des directeurs de la Société Générale avant la guerre. Fortement impliqué dans le développement des sports au sein de l'entreprise dans les années 1900, il appartient à cette nouvelle élite dirigeante du secteur bancaire qui a connu une ascension sociale remarquable. Il meurt en 1917 des suites d'une longue maladie. Cf. C. Ronzon-Belot, «Banquiers de la Belle Époque. Les dirigeants de la Société Générale vers 1900 », Histoire, économie et société, vol. 23, 2004, p. 411-432, et X. Breuil, Le Club Athlétique de la Société Générale. Histoire d'une succursale de champions, Saint-Cyrsur-Loire, Alan Sutton, 2008.

34. Archives de la Société Générale, B 1049, « Note sur l’activité du CASG Paris », mars 1925. 
du domaine autorise que « tous les jours et principalement les samedis et les dimanches, maris, femmes et enfants pourront venir se reposer, respirer le grand air et se distraire et au besoin faire du sport " ${ }^{35}$. Le stade ainsi conçu revêt une dimension globale en proposant par son étendue, sa configuration, ou ses aménagements, des activités qui s'adressent à un cercle bien plus large que les seuls pratiquants. La propension des dirigeants à diversifier les activités dans les stades qu'ils aménagent ne vise pas uniquement les familles de sportifs ${ }^{36}$. Les étangs du domaine de l'Association Sportive de la Bourse permettent le développement de loisirs comme le canotage, la pêche et la natation pour les membres non pratiquants : "Mais comme tous les Boursiers ne sont pas sportifs, et que l'effort consenti par la Chambre et les syndicats a pour but d'être également utile et agréable à tous les membres de la Bourse et leurs familles, il y aura le coin des jeux plus calmes (croquet, boules et un emplacement de jeux pour enfants $)^{37}$. " Alors que l'on pourrait penser que la création d'espaces spécifiques privilégie les terrains de sport à proprement parler, on remarque, lorsque l'étendue de l'espace et les ressources de l'association l'autorisent, que le principe de la diversification des installations est de mise. Le modèle en la matière est probablement le stade du club d'Enghien-Ermont, d'une superficie de deux hectares, qui comprend, outre le terrain de football, une piste de course à pied, un portique avec tous les agrès pour la gymnastique, six courts de tennis, un champ de tir au fusil et un de tir à l'arc, un terrain de boules, sans compter la salle d'armes, les salles de réunion, la buvette, le restaurant et la salle de jeux de billard regroupés dans un pavillon annexe ${ }^{38}$.

Cette diversification n'est pas l'apanage des clubs d'entreprise. En effet, dès les années 1910, les patronages catholiques qui bénéficient d'installations pérennes proposent un élargissement des activités susceptibles d'être pratiquées dans un complexe sportif. C'est ainsi que l'Étoile des Deux Lacs, patronage de la paroisse Saint-Honoré d'Eylau dans le XVI e arrondissement, dispose au domaine de la Marche d'un espace clos de treize hectares, résolument sportif et familial, avec ses trois terrains de football, sa piste d'athlétisme et quelques courts de tennis :

«Le sportif peut aller là-bas avec sa famille. Les parents trouveront dans le petit bois et aux alentours toutes les promenades agréables que l'on peut rêver dans un site comme Vaucresson. Si le papa adore la pêche, il aura un étang poissonneux. Si les parents ne veulent pas se promener, ils trouveront une salle de lecture et des jeux tranquilles, et s'ils n'aiment que le théâtre, la troupe des Deux Lacs jouera en plein air, dans le théâtre de verdure [... ${ }^{39}$."

Cette volonté de donner une empreinte familiale se retrouve dans d'autres patronages de la Seine, comme l'illustre le cas du Patronage Championnet, installé dans le XVII e arrondissement. En contractant un bail de location au Stade Dian de Villetaneuse, les dirigeants, au regard de l'investissement financier et de la proximité

35. Bulletin de l'Association Sportive de la Bourse, $1^{\mathrm{er}}$ janvier 1929.

36. On peut noter par ailleurs, comme le souligne Patrick Fridenson, que l'offre patronale s'est considérablement diversifiée et a pu, dans le monde de l'automobile, orienter la demande sociale des ouvriers : P. Fridenson, "Les ouvriers de l'automobile et le sport ", Actes de la recherche en sciences sociales, septembre 1989, p. 50-62.

37. Bulletin de l'Association Sportive de la Bourse, $1^{\mathrm{er}}$ mai 1928.

38. L'Auto, 13 août 1929.

39. Ibid., 19 mai 1922. 
du patronage, exigent de leurs joueurs de football « que vous et vos familles nous rendrez visite souvent et que, surtout, vous ne rechignerez pas devant la petite cotisation de un Franc qui vous sera réclamée à l'entrée " ${ }^{40}$. La notion de famille dans le cadre des patronages ne concerne pas seulement les conjoints et enfants des joueurs des équipes de football mais englobe également les parents des membres les plus jeunes. Cette conception tend à réduire les effets néfastes de la pratique sportive souvent accusée de diluer les liens familiaux par la multiplication des parties de football dominicales ${ }^{41}$.

Ainsi, l'acquisition et l'aménagement d'un espace clos, réservé aux membres de l'entreprise ou du patronage, est l'occasion de mettre en place un dispositif beaucoup plus large que celui nécessaire à la pratique. Le contrôle social à l'œuvre dans ces enceintes sportives institue le principe de l'extensivité. D'une part, en proposant aux côtés des espaces sportifs des aménagements périphériques centrés sur la détente et les loisirs, les dirigeants abrogent le temps court, compté, de la pratique sportive de l'employé pour le dilater à l'échelle de la journée. Les activités proposées, comme la pêche ou la pétanque, exigent un temps de pratique assez long et œuvrent ainsi à la sédentarisation des individus. L'existence d'une buvette permet d'envisager une présence durable des membres sur le site. D'autre part, le principe d'extensivité s'applique aux membres de l'entreprise qui échapperaient aux tentations de la pratique sportive voire aux membres de la famille du pratiquant. Il permet alors d'élargir le périmètre de recrutement social de l'espace sportif. Cette maximisation des profits sociaux du stade témoigne des préoccupations de certains dirigeants sportifs de l'entre-deux-guerres. En effet, les directeurs de patronage sont confrontés depuis la fin des années 1900 à l'autonomisation progressive des pratiquants et à la sécularisation des pratiques ${ }^{42}$. De même, les patrons doivent veiller à ce que leurs employés n'entrent pas dans les clubs affiliés à la fédération communiste ${ }^{43}$ qui, dans les années 1920 et surtout 1930, combat ouvertement les clubs sportifs patronaux. Dans cette perspective, l'intégration de la famille dans le stade renforce l'adhésion de l'individu à l'association suivant un processus de "violence symbolique ». C'est en effet au moment où les complexes sportifs s'érigent en espaces familiaux que se diffuse l'idée selon laquelle le club sportif, notamment le club d'entreprise, est une "grande famille». Ce discours, alimenté par les dirigeants eux-mêmes, est d'autant plus porteur et crédible qu'il se met en place à une époque où ceux-ci, non seulement essaient d'intégrer tous leurs employés à l'espace récréatif, mais encore associent le plus largement possible les membres de

40. Entre Nous, organe des auvres de garçons de la Paroisse Sainte-Geneviève des Grandes Carrières, 10 septembre 1933.

41. J. SOREZ, Footballs en Seine : histoire sociale et culturelle d'une pratique sportive dans Paris et sa banlieue de la fin des années 1880 à 1940, thèse d'histoire sous la dir. de J.-F. Sirinelli, IEP de Paris, 2011. Sur la relation entre pratique sportive et désagrégation des liens familiaux, on se reportera plus précisément au chapitre 6.

42. Y. Tranvouez, «Le sport catholique en France ", Vingtième Siècle. Revue d'histoire, 2006/4, n92, p. 171-180. Pour Yvon Tranvouez, l'autonomisation du football a lieu à partir des années 1920. Notre thèse montre que dans le département de la Seine ce processus est à l'œuvre dès la fin des années 1900. Cf. J. Sorez, Footballs en Seine..., op. cit., chapitre 1.

43. Les communistes avaient pris le contrôle de la Fédération sportive du travail (FST), qui avait succédé en 1919 à la Fédération socialiste des sports et gymnastique (FSSG) d'avant-guerre. Le congrès du 24 décembre 1934, constitutif de la Fédération sportive et gymnique du travail, marque l'aboutissement d'une réunification caractéristique du Rassemblement Populaire, dont la FSGT est l'une des composantes. 
la famille du joueur. Le transfert symbolique de la petite famille, composée principalement des membres du foyer conjugal, vers la grande, qui est le club et parfois l'entreprise, s'effectue de manière privilégiée dans ces enceintes associatives. Le stade est alors le vecteur essentiel de l'intégration de l'ordre domestique dans la structure plus large de l'ordre social. Par conséquent, le stade représente le lieu par excellence de l'incorporation de la notion d'une famille élargie et donc de domination sociale et permet de faciliter cette " construction de l'homologie " entre les structures de rapports familiaux et, pour notre étude, les rapports associatifs ${ }^{44}$.

Ce processus atteint son paroxysme dans les stades dits communautaires. En effet, au sein des communautés de migrants, provinciaux ou étrangers, qui ont le privilège d'en posséder un, le stade constitue un véritable point de fusion identitaire. L'Union Sportive des Bretons de Paris dispose dans les années 1930 d'un stade à Villejuif. À la mort de Joseph Le Brix, aviateur décédé en 1933 au cours d'un trajet reliant Paris à Tokyo ${ }^{45}$ et membre de l'association, le club des Bretons de Paris décide de donner son nom au stade ; trois ans plus tard, une souscription auprès de toute la communauté bretonne est ouverte pour l'érection d'une stèle ou d'un monument commémoratif. La souscription ne représente pas uniquement une célébration posthume d'un héros provincial, mais alimente la dimension communautaire de l'enceinte et de ce fait transforme le stade Joseph Le Brix afin qu'il " ne soit pas seulement le terrain de sports d'une société, mais la chose de tous les Bretons " ${ }^{46}$. Cette instrumentalisation identitaire de l'espace sportif est encore plus marquée dans le cas de la communauté suisse de Paris. Le stade de la Porte Dorée, obtenu grâce à l'intervention décisive de Charles Courvoisier-Berthoud ${ }^{47}$, est utilisé par l'Union Sportive Suisse pour toutes les compétitions de football auxquelles elle participe. Cependant, elle est aussi le lieu par lequel se cultive l'attachement à la mère-patrie dans la mesure où elle sert au mois d'août de cadre à la fête nationale suisse à Paris. Paré pour l'occasion de drapeaux cantonaux et du drapeau national, il réunit deux mille Suisses en août 1931 qui assistent à un match de football opposant deux équipes de l'U. S. Suisse organisé parmi de nombreuses manifestations en l'honneur de la Patrie. La dimension patriotique du stade est exploitée par le président d'honneur de l'association, l'ambassadeur de Suisse à Paris, qui a pour habitude d'y prononcer un discours ${ }^{48}$. Lieu fédérateur de l'associationnisme suisse à Paris, le stade renforce la capacité de l'association sportive à mobiliser le sentiment d'appartenance à la communauté. Lorsque le stade est menacé de disparaitre en juin 1932, le Journal Suisse de Paris tente de réactiver le patriotisme à l'œuvre au cours de ces manifestations pour sauver cet espace de communion communautaire :

"Le stade suisse est devenu pour les Suisses de Paris une sorte de terre sacrée où les appellent les fêtes vouées au culte de la Patrie. C'est là que nous nous sentons

44. Rémi Lenoir montre que les différentes formes de pouvoir, notamment politique, s'approprient et incorporent la notion de famille pour devenir légitimes et se transmettre à la manière d'un patrimoine, tout en redéfinissant sans cesse leur contenu pour l'adapter aux exigences du maintien de leur rang : R. LENOIR, "Famille et politique : les métaphores familiales de l'ordre politique ", Regards Sociologiques, $\mathrm{n}^{\circ} 15,1998$, p. $7-14$.

45. Match, 15 septembre 1931.

46. Armor, revue mensuelle illustrée de défense des intérêts bretons, 15 février 1936.

47. Charles Courvoisier-Berthoud, banquier, est un des principaux évergètes de la colonie suisse de Paris. Il est également trésorier de la Chambre de commerce suisse de Paris à sa fondation en 1918.

48. Le Journal Suisse de Paris, 7 août 1931. 
le mieux en harmonie pour commémorer le $1^{\text {er }}$ août et chanter en commun notre pays bien aimé. Ce serait nous amputer volontairement d'un lambeau de la terre helvétique que de consentir à la disparition d'une telle œuvre ${ }^{49}$. "

Au demeurant, plus encore qu'un espace de fusion identitaire, le stade communautaire est avant tout le lieu de la reconnaissance et de la réaffirmation du pouvoir légitime institué, que ce soit en raison de la place prépondérante des autorités officielles ou par l'association de notables de l'élite économique suisse à Paris.

\section{Le stade municipal et la " disciplinarisation " du corps social}

Entre la fin du XIX ${ }^{e}$ siècle et les années 1920, le football dans la Seine demeure une pratique relativement marginale et a du mal à s'installer dans des espaces de pratique spécifiques et pérennes. Le nomadisme des clubs de football explique la nécessité pour les joueurs de monter et démonter leurs cages de but et de les ranger dans un lieu sûr après la rencontre ou d'utiliser la remise d'un commerce de bouche pour s'habiller. Il implique aussi l'absence d'aménagements pour le public tels qu'une tribune ou même une barrière entourant le terrain. Si le vol et la dégradation de matériel sont monnaie courante dans les premières décennies de la pratique, la configuration spatiale rudimentaire du terrain de football est surtout à l'origine d'une interaction prononcée entre les joueurs et les spectateurs. D'une part, les joueurs se montrent très sensibles aux cris qui accompagnent les péripéties des rencontres sportives et il n'est pas rare que le déroulement des matchs en soit affecté. Ils doivent aussi composer avec le corps du spectateur, qui en l'absence de démarcation matérielle contraignante, est complètement immergé dans la partie de football. Que ce soit à la mi-temps des matchs pour donner des conseils aux joueurs, au cours du match pour désapprouver le jeu dur de l'équipe adverse ou pour prêter main-forte dans une rixe, le public, principalement composé de proches des acteurs du match et de joueurs d'autres associations, envahit régulièrement le terrain.

La multiplication des enceintes sportives municipales durant l'entre-deux-guerres transforme considérablement ces usages inhérents aux parties de football. D'un côté, le football devient l'élément central des stades en même temps qu'il assoit sa pérennité en s'imposant dans des espaces exclusivement dédiés à la pratique sportive. De l'autre, la portée sociale du football en est profondément transformée. Tant que sa pratique était nomade, qu'elle concernait peu de spectateurs et que son implantation ne relevait que des dirigeants généralement éphémères du monde associatif, le comportement des différents acteurs des rencontres de football restait confiné à l'entre-soi sportif. Mais dès lors que le football entre dans un stade, qui plus est, édifié et géré par une institution politique, il se place sous l'autorité d'un pouvoir plus légitime que le pouvoir associatif et surtout plus contraignant.

Tout d'abord, la plupart des municipalités qui font bénéficier les sociétés sportives d'un stade ou d'un complexe sportif édictent un règlement intérieur qui établit une situation contractuelle entre les usagers et la commune ${ }^{50}$. À l'image des règlements

49. Ibid., 17 juin 1932.

50. La rédaction des règlements de stade est le fait d'élus locaux et de dirigeants du monde associatif dans le cadre de commissions des sports municipales ou d'offices municipaux des sports lorsqu'ils existent. Toutefois, si le monde associatif est représenté, il n’a en général que peu de poids face aux élus dans les délibérations que préside bien souvent un adjoint quand ce n’est pas le maire en personne. 
d'usine qui se mettent en place avec le développement industriel au XIX ${ }^{\mathrm{e}}$ siècle et représentent " la forme concrète du pouvoir et la légitimation idéologique de celui-ci " ${ }^{51}$, les règlements de stade illustrent l'un des ressorts essentiels de la politique mayorale. La préoccupation centrale à l'origine de cette réglementation est la réduction des nuisances liées à l'activité sportive. Ainsi l'Union Sportive de Gennevilliers, qui obtient un bail de location pour neuf années sur un terrain municipal de $11000 \mathrm{~m}^{2}$ en 1927, est-elle mise dans l'obligation "de le clôturer, d'entretenir la clôture et prendre toutes les dispositions utiles pour que les balles et ballons ne puissent en aucun cas tomber en dehors du terrain ". Par ailleurs, elle doit prendre " toutes les dispositions utiles afin de dérober à la vue des passants la toilette et les douches des joueurs " ${ }^{52}$. De même, l'article 14 du règlement du stade de Clichy stipule que "les athlètes se déshabilleront uniquement dans les vestiaires réservés à cet effet " ${ }^{53}$. Ces règlements s'appuient sur l'énumération de comportements sociaux jugés déviants. C'est ainsi que " toute tenue négligée et toutes manifestations extérieures indignes d'un esprit sportif (ivresse, injures, rixes), seront sévèrement réprimées et toute récidive pourra entraîner l'exclusion du fautif du Stade municipal » de Clichy. De même, à Saint-Denis, la plus grande correction est recommandée au cours des matchs ou compétitions : " toute personne (joueur, soigneur, spectateur, etc.) qui par ses gestes ou ses paroles aurait une attitude grossière ou injurieuse à l'égard d'autres personnes, quelles qu'elles soient, pourra être exclue séance tenante par le gardien du stade " L'extrême rigueur des règlements tient en partie au fait que le stade communal est un espace sous contrôle municipal, engageant à ce titre la réputation de la commune et la responsabilité de ses édiles, comme l'atteste l'article $18 \mathrm{du}$ règlement du stade municipal de Saint-Denis, aux termes duquel les sociétés locales doivent accorder aux équipes visiteuses " une hospitalité digne de ce nom " 55 .

La prise de contrôle sur les individus est confirmée par l'arsenal répressif mis en place par ces règlements. Alors que les clubs de football restent aux yeux des fédérations sportives les garants de l'ordre et du bon déroulement des épreuves, les règlements des stades communaux montrent la manière dont l'autorité municipale vient se substituer à l'autorité associative. Certes, cette dernière conserve une partie de ses prérogatives de police sur les terrains, mais elle est dans bien des cas déléguée à la société d'exploitation du stade ou à l'organisme municipal des sports. À Aubervilliers, c'est la société du stade municipal, dont le siège social se trouve à la mairie qui, outre l'encouragement à la pratique des sports et de la culture physique, " a pour but la gestion, la surveillance, et l'entretien du stade municipal " ${ }^{56}$. Le dessaisissement de l'association est aussi palpable dans le règlement de Clichy où, " dans le but de faire respecter le présent règlement, la municipalité ou ses délégués auront le droit de pénétrer dans tous les endroits où il y aura lieu d'exercer le contrôle et la surveillance " 57 ,

51. A. Melucci, «Action patronale, pouvoir, organisation. Règlements d'usine et contrôle de la main-d'œuvre au XIX' siècle ", Le Mouvement Social, n97, octobre-décembre 1976, p. 139-159.

52. AM Gennevilliers, R 99/4, sociétés sportives "Union Sportive de Gennevilliers (1905-1936) ".

53. AM Clichy, 3R 2, règlement intérieur du stade municipal, délibérations du 27 avril 1927.

54. AM Saint-Denis, 6M 15, construction du stade du Barrage (1932-1939).

55. Ibid., projet de règlement du stade municipal de Saint-Denis daté du 27 février 1939 et approuvé en conseil municipal ce jour.

56. Ibid., exemplaire du règlement du stade municipal d'Aubervilliers (s. d.).

57. AM Clichy, 3R 2, règlement intérieur du stade municipal, délibérations, art. 10. 
alors qu'à Saint-Denis, le secrétaire administratif de l'office municipal des sports est habilité à réunir, en cas d'incidents, une commission qui instruira un dossier remis au maire, lequel pourra prendre les sanctions jugées utiles ${ }^{58}$.

Les attitudes corporelles des individus constituent la cible privilégiée des règlements municipaux destinés aux différents usagers dont les comportements déviants sont soigneusement catalogués. À la manière des traités de civilité, on peut penser qu'ils participent à la tentative de normalisation des manières de se comporter sur un stade, tant sur le terrain que dans les tribunes. À la pudeur des corps qui doivent se dérober à la vue des passants par l'usage du vestiaire, l'attention portée à la mesure des gestes et à la correction du vocabulaire de tous les acteurs du champ sportif local sont la trace d'une emprise progressive du pouvoir municipal sur les comportements sociaux de leurs concitoyens. En mettant à disposition un stade communal qu'elle a aménagé et dont elle gère l'utilisation, la municipalité impose une normalisation des comportements qui agit dans le sens d'une régulation de la violence, sur le terrain et en dehors.

Ces prescriptions à valeur normative nous montrent que le stade s'inscrit dans l'ensemble des espaces de contrôle social qui font du corps le médiateur privilégié du pouvoir associatif, patronal ou municipal. Néanmoins, si l'observation des prescriptions laisse supposer qu'il y a bien une instrumentalisation sociale et politique du stade, on ne peut négliger les spécificités et les objectifs divergents de ces acteurs. Les directeurs des ouvres catholiques, tout comme les patrons, tentent, par la jouissance et le contrôle des espaces de pratique et de sociabilité sportives, d'intensifier le contrôle qu'ils exercent sur leurs membres ou employés. Par le sport, ils œuvrent au renforcement d'une sociabilité d'entreprise ou paroissiale parfois précaire ${ }^{59}$. En revanche, dans les municipalités socialistes et communistes, le stade s'inscrit dans une politique plus large d'organisation des loisirs de la classe ouvrière. Il est davantage pensé comme un vecteur d'émancipation de ces organisations sociales dénoncées comme conservatrices. Il est dans ce cas un des espaces privilégiés de l'" hyperpolitisation du quotidien ${ }^{60}$. Ainsi, durant les grèves de 1936, de nombreuses municipalités communistes tentent d'organiser des rencontres sportives visant à financer le manque à gagner des grévistes et font du stade un espace de mobilisation politique ${ }^{61}$.

Cette volonté de déterminer minutieusement ce qu'il est possible de faire sur un stade est aussi le reflet de la trajectoire sociale du football. Sa pratique connaît dans l'entre-deux-guerres une diffusion sociale plus large que dans ses périodes de recrutement précédentes, notamment à partir du milieu des années 1930 où les clubs sportifs de la FSGT, fédération sportive ouvrière réunifiée, attirent un nombre croissant d'ouvriers ${ }^{62}$. De même, le renouvellement opéré par la saignée démographique

58. AM Saint-Denis, 6M 15, construction du stade du Barrage (1932-1939), règlement du stade municipal de Saint-Denis du 27 février 1939.

59. Il s'agit notamment de fixer ou d'attirer une main-d'œuvre parfois volatile pour le patronat ou de se rapprocher du prolétariat urbain longtemps négligé par les œuvres catholiques.

60. M. Fontaine, "Travail et loisirs ", in J.-J. Becker et G. CAndar (dir.), Histoire des gauches en France, vol. 2, Paris, La Découverte, 2004, p. 709.

61. J. Sorez, Footballs en Seine..., op. cit., chapitre 5.

62. Sur les vicissitudes du sport ouvrier, on se reportera aux travaux d'A. Gounot, notamment "Sport ouvrier et communisme en France, 1920-1934 : une rencontre limitée ", Stadion. International Journal of History of Sport, vol. 23, 1997, p. 83-95 et, sur l'élan du football travailliste des années 1930, à N. Ksiss, « Le mouvement ouvrier balle au pied, culture populaire et propagande politique : l'exemple 
de la Grande Guerre et la nouvelle attention portée à l'éducation sportive de la jeunesse opèrent une "démocratisation " de la pratique du football. Dès lors, la réglementation et le contrôle social qu'elle impose traduit la coexistence sur l'espace communal de sociétés aux profils institutionnels et sociologiques différents. À SaintDenis, l'hétérogénéité des sociétés bénéficiaires du terrain de football est à l'origine d'un règlement tout aussi contraignant que celui de Clichy. Il est le fruit d'une négociation entre d'un côté le Football club athlétique dionysien, l'Athletic club espagnol, les Gaziers de Paris affiliés à la Fédération française de football association (FFFA), et de l'autre le Club sportif ouvrier dionysien (FSGT). Les discussions ont pour but de faire renoncer le CSOD au cri de "Sport rouge! " pendant les rencontres sportives, qui suscite l'indignation des dirigeants et joueurs des autres associations. Elles aboutissent à l'adoption d'un texte très normatif puisque "seule l'exclamation conventionnelle dans les milieux sportifs "hip, hip, hip, hurrah !" " est autorisée ${ }^{63}$.

Enfin, la surveillance du stade est, dans ce processus de domestication des comportements, un enjeu de taille. C'est pourquoi le choix et l'engagement d'un gardien deviennent primordiaux dans la gestion des équipements sportifs, à l'image des portiers de manufacture ou des gardiens d'usine qui étaient chargés de surveiller les entrées et sorties au sein des espaces de production industrielle. Le gardien est non seulement le garant de l'intégrité matérielle des infrastructures, mais il a surtout en charge le contrôle du comportement des usagers. Le gardien du stade de Clichy, M. Saussais dit André, ancien concierge du stade de l'Union Sportive Athlétique de Clichy, s'occupe, " au-delà de la propreté des lieux, de la surveillance de jour et de nuit, notamment le dimanche où il assure "une surveillance active" autour des vestiaires et aucun vol n'a été signalé dans les comptées officielles, aux dits vestiaires ${ }^{64}$. Il garde aussi les ballons des divers groupements et les remet en état, pose les filets et trace le terrain. Organisateur, il fait respecter le calendrier et est aussi responsable de la perception des entrées ${ }^{65}$. De même, le cantonnier-gardien du terrain municipal de Bagatelle met à la disposition des équipes les cages des buts et peut à tout moment contrôler les sociétés qui doivent se munir d'une fiche validée par la sous-commission des terrains mentionnant le jour, l'heure et le numéro du terrain ${ }^{66}$. Les édiles municipaux privilégient pour la charge de gardien de stade des individus de confiance, comme l'attestent l'existence de rapports d'activité et la lettre de recommandation des dirigeants de l'Union sportive athlétique de Clichy, alors dépossédée de son stade par la municipalité, pour obtenir du maire le maintien à son poste de celui qu'ils avaient choisi. Rémunérée 3000 francs par an à Clichy, la fonction de gardien comportait quelques dangers puisque celui du stade de Clichy est arbitre de la Fédération française de boxe et surtout sportif pratiquant ${ }^{67}$, alors que les deux gardiens de La Courneuve, suite à des vols qui ont eu lieu dans le Parc,

du football travailliste en région parisienne (1908-1940) ", Cahiers d'Histoire, revue d'histoire critique, $\mathrm{n}^{\circ} 88,2002$, p. 93-104.

63. AM Saint-Denis, 6M 15, construction du stade du Barrage 1932-1939, procès-verbal de la réunion du 16 décembre 1938.

64. AM Clichy, 3R 2, stade municipal, rapport de l'administrateur municipal du stade du $1^{\text {er }}$ février 1928 sur le gardien.

65. Ibid., lettre du maire de Clichy au préfet de la Seine (s. d.).

66. AD Seine, 1304W, commission des terrains de jeux de Bagatelle, règlement intérieur de 1928 pour l'utilisation des terrains de jeux et de la pelouse.

67. AM Clichy, 3R 2, stade municipal, lettre du maire de Clichy au préfet (s. d.). 
expriment leur désir d'obtenir un fusil ${ }^{68}$. Le zèle mobilisé dans l'exécution de leur mission se révèle sans doute parfois un peu excessif, à l'image du témoignage que publie L'Humanité sur des incidents qui se sont produits au stade de Clichy en avril 1934. Alors que la municipalité refusait l'accès au club de la Fédération Sportive du Travail (FST) de la commune, ce dernier a bravé l'interdit en faisant pénétrer une trentaine de membres dans le vestiaire, profitant de l'absence du gardien-administrateur du stade et secrétaire de la Prolétarienne sportive clichoise qui, alerté, " arriva et dans les insultes, alla jusqu'à dire au président du CPSC qu'il "lui foutrait une balle dans la peau, qu'il en avait déjà descendu deux, qu'il en descendrait bien un troisième" ${ }^{69}$."

À partir des éléments que nous venons d'évoquer, on peut estimer que les stades reposent sur deux types différents de contrôle social ${ }^{70}$. D'une part, certains espaces correspondent à un " contrôle de type municipal ", où l'accès est ouvert à un grand nombre de pratiquants anonymes, et dont l'usage s'appuie sur une relation contractuelle entre le sportif et l'autorité sociale qui l'administre, matérialisée par la production d'un règlement écrit. Ce modèle, en explicitant les comportements déviants et en énumérant les sanctions éventuelles, relève de la surveillance et de la punition immanente et extérieure. D'autre part, un " contrôle de type patronal ", présenté sous les traits d'une mise à disposition bienfaitrice des installations sportives, fonde sa relation aux usagers sur l'incorporation d'une discipline intérieure. Parce que le stade est la famille, il est intrinsèquement lié à la notion d'autorité et de respectabilité. Ce modèle assied son autorité sur des modes de contrôle intériorisés, beaucoup plus contraignants que l'édiction réglementaire. Ce n'est d'ailleurs pas un hasard si les institutions sociales conservatrices comme les patronages catholiques ou le patronat aux attentions paternalistes ont privilégié l'intériorisation des contraintes sociales.

Pour l'entre-deux-guerres, peu d'archives nous permettent de mesurer l'impact réel de cette mainmise sur la pratique et les pratiquants. Au regard des campagnes menées contre les brutalités commises sur les terrains de football par les différentes institutions sportives dans les années 1930, il semble que l'ordre municipal pas plus que l'ordre patronal ne résiste totalement aux enjeux et à la rivalité sportifs ${ }^{71}$. Surtout, si le stade sert et manifeste l'autorité sociale ou politique, il est également un espace de contestation et de transgression. Pour preuve, les nombreuses manifestations sportives organisées par les fédérations socialistes et communistes à la fin des années 1920 et surtout dans les années 1930 contre le fascisme et la guerre, au cours desquelles le drapeau communiste était déployé, l'Internationale chantée et le gouvernement contesté, ou encore les matchs de football organisés par les ouvriers grévistes durant le Front Populaire sur les terrains jouxtant les usines ${ }^{72}$.

68. AM La Courneuve, 4R 1, Parc des Sports, sous-commission du Parc des Sports, séance du 19 janvier 1934.

69. L'Humanité, 11 avril 1934.

70. Pour une réflexion synthétique mais stimulante sur la notion de contrôle social, on se reportera à R. Lenoir, "Contrôle social. La construction d'une notion et ses enjeux ", Informations sociales, août 2005, p. 6-15.

71. Paris Football, l'organe de la Ligue Parisienne de Football Association, publie régulièrement des annonces demandant explicitement à ses joueurs de jouer sans brutalité, tout comme Sport, revue de la FSGT.

72. Cf. Archives nationales (AN), Paris, $\mathrm{F}^{7}$ 13137, et AN, Fontainebleau, 20010216, art. 139 et 140. 
Enfin, les témoignages de certains contemporains nous laissent supposer que la pratique sportive dans les enceintes patronales n'excluait pas la conscience ouvrière de cette mainmise. Ainsi, la réponse de Marcel Donati, fils d'immigré italien et ancien ouvrier lamineur à Usinor-Rehon, qui, interrogé sur les modalités de la présence paternaliste, exprime toute la lucidité des ouvriers sur ces dispositifs de contrôle :

" À travers tout. Par exemple, le stade, le terrain de sports. C'était le stade Arthur-Cousin, du nom de l'ancien directeur de l'usine. Le stade appartenait à l'usine. La salle de cinéma, qui était aussi la salle des fêtes, appartenait à l'usine. La bibliothèque appartenait à l'usine. On sentait l'usine en permanence. Ce n'était pas la présence physique de la direction, c'était la présence de l'usine, elle était partout. ${ }^{73}$

Cette évocation spontanée de l'instrumentalisation patronale des installations sportives nous montre que les pratiquants avaient pleinement conscience du rôle que jouait le stade dans les rapports sociaux. Elle nous suggère aussi que l'homologie entre l'usine et le stade ne s'arrête pas à la proximité spatiale, ni aux seules conditions de pratiques ouvrières dans le cadre patronal. On peut émettre l'idée selon laquelle la constitution de l'espace sportif, qui passe des terrains vagues, sommairement aménagés et souvent mal desservis, à l'espace réglementé et organisé du stade est, par de nombreux aspects, similaire à celle de l'espace industriel. D'abord marqués par une conquête anarchique de l'espace environnant, les sites de production n'ont au départ aucune clôture et jouxtent des terrains agricoles et des zones résidentielles ${ }^{74}$. Ce n'est qu'à partir du début du XX $\mathrm{X}^{\mathrm{e}}$ siècle que leur clôture et la rationalisation de leur organisation se mettent progressivement en place. Si la trajectoire spatiale de la pratique sportive suit une logique similaire avec un décalage décennal, les principes de surveillance et d'interdépendance des espaces industriels sont aussi caractéristiques des enceintes sportives. En effet, tant dans les usines à partir du début du $\mathrm{XX}^{\mathrm{e}}$ siècle que dans les stades, on constate la multiplication des installations destinées à améliorer le confort des travailleurs ou usagers. Comme le souligne Michelle Perrot, ces améliorations ont pour corollaire la multiplication des interdits, processus dont témoignent également les aménagements et la réglementation des stades ${ }^{75}$. Reste que la présence au stade diffère considérablement de celle des lieux de production puisque le temps de pratique sportive, en dépit des efforts pour en prolonger la durée par la diversification des activités et des installations, est moins contraignant. Il faudrait toutefois sonder si le stade dans les différents dispositifs de contrôles sociaux n'est pas d'autant plus efficace que les individus, joueurs comme spectateurs, s'y rendent de leur plein gré. Cette piste permettrait alors d'expliquer que, dans la crise actuelle des espaces d'enfermement, le stade a encore de beaux jours devant lui.

73. Interview de Marcel Donati, ouvrier lamineur à Usinor-Rehon, militant CGT (30 septembre 1985), propos recueillis par Marianne Debouzy, Le Mouvement Social, $\mathrm{n}^{\circ} 144$, juillet-septembre 1988, p. $37-49$, p. 39.

74. G. NoIriel, Longwy : immigrés et prolétaires (1880-1980), Paris, Presses Universitaires de France, 1984, p. 137.

75. M. Perrot, "De la manufacture à l'usine en miettes ", Le Mouvement Social, nº 125, L'espace de l'usine, 1983, p. 6. 